\title{
An Optimal Proportional Integral Design for a practical Controlled s
} m.m nassar, M.M Fouad, G.M Samy

\begin{abstract}
A practical study of a fluid system including Benfield solution (B.S.) in a group of containers is presented. The operating function of the coupling valves and level transmitters inside the containers are obtained. The present work measures the practical values of the quantity and level of B.S where different techniques of optimization are utilized to minimize the error performance nsing proportional integral control parameters (PI) to maintain the level of B.S constant inside the container. The experimental studies and the theoretical analysis are carried with proper results
\end{abstract}

